\title{
A Simple Direct Microcontroller Interface for Capacitively-Coupled Resistive Sensors
}

\author{
Lakshmi Areekath ${ }^{1}$, Student Member, IEEE, Boby George ${ }^{1}$, Senior Member, IEEE, and Ferran Reverter ${ }^{2}$ \\ ${ }^{1}$ Department of Electrical Engineering, Indian Institute of Technology Madras, Chennai, India. Email:ee17d014@smail.iitm.ac.in \\ ${ }^{2}$ Department of Electronic Engineering, Universitat Politècnica de Catalunya, Barcelona, Spain. Email: ferran.reverter@upc.edu
}

\begin{abstract}
A novel approach to interface capacitively-coupled resistive sensors directly to a microcontroller is presented in this paper. The existing schemes for the measurement of the resistance of a sensing element that is inaccessible, unless through capacitive coupling, are complex. This paper presents a direct microcontroller interface solution for this category of sensors. When a resistive sensor is coupled to the measurement system through capacitances, one of the main challenges is to make the resistance measurement insensitive to variations in the coupling capacitances. This is achieved in the proposed direct microcontroller approach, without using the synchronous demodulation technique adopted by conventional approaches. Additionally, the output is independent of the value of the charging capacitor, the supply voltage and the preset threshold voltage. The feasibility study of the proposed scheme has been conducted in two steps by building suitable prototypes. The test results from the initial study, in which the experimental setup was designed in such a way that its performance was not limited by the non-idealities of the microcontroller, showed a maximum error of $0.28 \%$. The subsequent study with the microcontroller interface exhibited a maximum error of $1.48 \%$. Thus, the accuracy of the proposed system is quite promising, with a measuring time of a few milliseconds. Higher accuracy can be achieved by using a microcontroller with higher time resolution, and a comparator with lesser delay and noise. This scheme does not use any expensive parts and can hence realize a highly practical, low-power and low-cost measurement system.
\end{abstract}

Keywords-interfacing circuit, resistive sensor, capacitive sensor, capacitive coupling, direct microcontroller interface

\section{INTRODUCTION}

Sensors play a key role in fueling progress in the fields of engineering, science, and industry. Their applications are evergrowing. Among these, resistance and capacitance-based sensors are used extensively in consumer electronics, automation, and automobiles. Resistive sensors are, for instance, used in the measurement of pressure [1], force [2], [3] and temperature [4], and in touchpads [5] and tactile sensing [6]. However, the conventional methods to measure the resistance of a resistive sensing element require direct contact of that element to the measurement circuit, thereby limiting the scope of its application and introducing complexity in its manufacturing process and the design of the sensor unit. In addition, the reliability of these methods is limited as direct contact can be critical. Capacitive sensors, on the other hand, have much lesser limitations with regard to these aspects. Yet, often they need complex signal conditioning to achieve output insensitivity to variations in the environmental parameters, like humidity, parasitic elements [7] and interference [8]. Some interesting applications of capacitive sensors include tactile [9], level [10] and humidity [11] monitoring. The complexity associated with signal conditioning required for resistive sensors is relatively less compared to that for capacitive sensors, as they possess higher selectivity to the sensing parameters of interest.

Juxtaposing the performance of the resistive and capacitive sensing paradigms brings forth the desirable possibility of having a sensor that combines the strengths of both. This occurs, for instance, in capacitively-coupled resistive sensors, where the information of interest is in the resistance but there is no direct contact with the sensing element. Such an approach can bring in improved performance and reduced complexity in manufacturing the sensor [12]. Potential applications of this type of sensor are the following. 1) A resistive touchpad wherein the change in resistance is measured through a capacitively-coupled arrangement. This could significantly reduce the complexity in the manufacturing and final assembly, and also increase the reliability as direct contact to the sensing element is eliminated. If a less complex measurement mechanism can be developed for such sensors using low-cost systems, it will even help scale the usage of new types of touch tracking pads that utilize resistive and capacitive sensing elements [5]. 2) A liquid conductivity sensor [13] using capacitively-coupled electrodes [12]. However, the circuit in [12] is relatively complex with the use of sinusoidal excitation and a phase-sensitive detector. One way to simplify the measurement scheme is to develop a direct microcontroller interface for such capacitively-coupled resistive sensors. Such an interface will help to realize a low-cost and reliable measurement system that consumes less power and thus, enables wide-spread use of this type of sensors.

Direct microcontroller interfaces have been developed for the measurement of resistance [14], [15], capacitance [16], and inductance [17], [18]. Novel calibration methods to reduce the measuring time of these circuits have also been presented in [19]. In such circuits, the microcontroller excites a passive RC or RL network and then measures the length of the transient response through an embedded digital timer. The main advantage is that no active electronic components are used between the sensor and the microcontroller, which reduces cost, size and power consumption of the design. The charge and discharge cycles in each of these cases are realized by programming the appropriate signals at the digital input-output pins of the microcontroller. However, none of the abovementioned circuits are suitable for the measurement of the capacitively-coupled resistive sensors described in the previous paragraph. 
In this paper, a simple direct microcontroller interface for capacitively-coupled resistive sensor is presented. The proffered scheme is independent of the values of the coupling capacitors, the charging capacitor, the power supply voltage, and the preset threshold voltage. It is simple in design, consumes less power, and carries out the measurement in a few milliseconds. This scheme does not use any expensive parts and can hence realize a low-cost measurement system.

\section{DIRECT $\mu$ C INTERFACE FOR CAPACITIVELY-COUPLED RESISTIVE SENSOR}

A block diagram of the proffered scheme is shown in Fig. 1. In Fig. 1, $R_{x}$ represents the resistive sensor element. It is coupled through the coupling capacitors $C_{1}$ and $C_{2}$. As illustrated, $R_{x}, C_{1}$ and $C_{2}$ are in series, and the nodes ' $a$ ' and ' $b$ ' are accessible for the direct connection to the microcontroller $(\mu \mathrm{C})$. The scheme uses a reference resistor $R_{\text {ref }}$, and a fixed (charging) capacitor $C$. As illustrated in Fig. 1, the microcontroller uses four of its digital input/output pins $\mathrm{D}_{\mathrm{IO}-1}, \mathrm{D}_{\mathrm{IO}-2}, \mathrm{D}_{\mathrm{IO}-3}$ and $\mathrm{D}_{\mathrm{IO}-4}$, the analog input pin $\mathrm{A}_{1}$, the built-in timer and comparator, to perform the measurement.

The two terminals of $R_{r e f}$ are connected to the digital pins $\mathrm{D}_{\mathrm{IO}-1}$ and $\mathrm{D}_{\mathrm{IO}-2}$, respectively. The end of $R_{\text {ref }}$ that is connected to $\mathrm{D}_{\mathrm{IO}-2}$ is connected to node ' $a$ '. This node is connected to $C_{1}$ which is one of the coupling capacitors to the resistive sensing element $R_{x}$. The effective capacitance $C_{12}$ due to $C_{1}$ and $C_{2}$, $C_{12}=\left(C_{1} C_{2} /\left(C_{1}+C_{2}\right)\right)$. One of the ends of $C_{2}$ is accessible at node ' $b$ '. Capacitor $C$ is connected to node ' $b$ ' to form the $\mathrm{RC}$ circuit that enables the measurement of $R_{x}$ in this direct- $\mu \mathrm{C}$ approach. The other terminal of $C$ is connected to ground. The node ' $b$ ' is also connected to $\mathrm{D}_{\mathrm{IO}-3}$ and $\mathrm{A}_{1}$, as given in Fig. 1.

To estimate $R_{x}$, the proposed scheme is operated in two modes - namely, mode $-R_{x}$ and mode $-\left(R_{\text {ref }}+R_{x}\right)$. The measurement sequence starts with mode $-R_{x}$, followed by mode - $\left(R_{r e f}+R_{x}\right)$. To execute this, the state of the pins $\mathrm{D}_{\text {IO- }}$ 1, $\mathrm{D}_{\mathrm{IO}-2}$ and $\mathrm{D}_{\mathrm{IO}-3}$ are varied as per Table-I, where LOW refers to $0 \mathrm{~V}$ or ground, $\mathrm{HIGH}$ to the de voltage $V_{D D}$, and $\mathrm{HZ}$ indicates the high-impedance state. $\mathrm{D}_{\mathrm{IO}-3}$ is in $\mathrm{HZ}$ state during the charging and discharging cycles. A timing diagram of the measurement process is illustrated in Fig. 2. The measurement begins when a start pulse (at the high to low transition) is provided at pin $\mathrm{D}_{\mathrm{IO}-4}$. Then, the $\mu \mathrm{C}$ sets $\mathrm{D}_{\mathrm{IO}-1}, \mathrm{D}_{\mathrm{IO}-2}$ and $\mathrm{D}_{\mathrm{IO}-3}$ to $0 \mathrm{~V}$, to discharge any residual charges that may be present in the capacitors. This is indicated as state '0' in Fig. 2. Following this, both $\mathrm{D}_{\mathrm{IO}-1}$ and $\mathrm{D}_{\mathrm{IO}-2}$ are set to HIGH. This is indicated as state ' 1 ' in Fig. 2. In this charging-phase, during mode $-R_{x}, R_{x}$ alone is present in the $\mathrm{RC}$ circuit. This is under the assumption that the value of output resistance of the digital pin, $R_{\text {pin }}$, is negligible compared to $R_{x}$. In this condition, $C$ is charged to the final voltage $V_{C F}$ given by (1). The measurement unit is in state ' 1 ' for a preset time $T_{C h 1} \cdot T_{C h 1} \gg\left(R_{x} C_{e q}\right)$, where $C_{e q}=C_{12} C /\left(C_{12}+C\right)$.

$$
V_{C F}=V_{D D} \frac{C_{12}}{C_{12}+C}
$$

As soon as the time $T_{C h 1}$ is over, the state ' 1 ' ends and the state '2' begins, as in Fig. 2. In state '2', both $\mathrm{D}_{\mathrm{IO}-1}$ and $\mathrm{D}_{\mathrm{IO}-2}$ are set

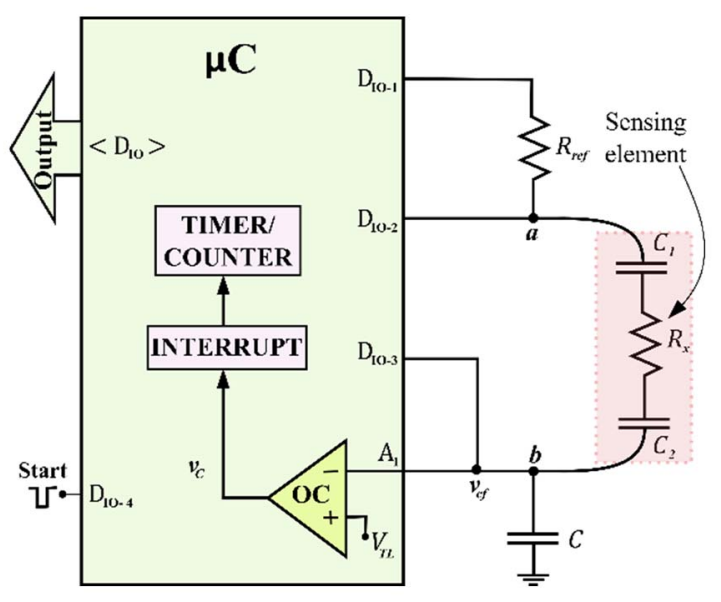

Fig. 1. Block diagram of the proposed direct microcontroller interface.

TABLE I: STATES OF THE DIGITAL PINS.

\begin{tabular}{|c|c|c|c|}
\hline \hline State & D $_{\text {IO--1 }}$ & D $_{\text {IO-2 }}$ & D $_{\text {IO-3 }}$ \\
\hline \hline 0 & LOW & LOW & LOW \\
1 & HIGH & HIGH & HZ \\
2 & LOW & LOW & HZ \\
3 & LOW & LOW & LOW \\
4 & HIGH & HZ & HZ \\
5 & LOW & HZ & HZ \\
6 & LOW & LOW & LOW \\
\hline \hline
\end{tabular}

to LOW, causing $C$ to discharge through $R_{x}$. In this discharging phase, the voltage $v_{c f}$ across $C$ is compared with an internal preset threshold voltage $V_{T L}$, as illustrated in Fig. 2. This is accomplished by feeding $v_{c f}$ to the input of the built-in comparator, $\mathrm{OC}$, via $\mathrm{A}_{1}$ as shown in Fig. 1. When the discharge occurs through $R_{x}$ alone (mode $-R_{x}$ ), the time interval $T_{1}$ taken for $v_{c f}$ to reduce from $V_{C F}$ to $V_{T L}$ is measured by the built-in timer, which starts counting when the discharge begins and stops when $v_{c f}=V_{T L}$ (via interrupt to timer from OC output $\left.v_{O C}\right)$. While discharging, during state '2', (2) can be written when $v_{c f}=V_{T L}$. It is assumed that $V_{C F}>V_{T L}$. This can be ensured, in practice, by selecting a value of $C$ such that $\left(C_{e q} / C\right)>1 / 2$, and setting $V_{T L}<<V_{D D} / 2$.

$$
V_{T L}=V_{C F} e^{-T_{1} / R_{x} C_{e q}}
$$

From (2),

$$
T_{1}=R_{x} C_{e q} \ln \left(\frac{V_{C F}}{V_{T L}}\right)
$$

The measurement unit is in state ' 2 ' for a preset time $T_{D h 1}$, where $T_{D h 1}=T_{C h 1}$. If we compute $R_{x}$ using (3), its accuracy will depend upon the accuracy in the measurement of $C_{e q}, V_{C F}$ and $V_{T L}$. To achieve an output independent of these parameters, another mode is introduced. Once state ' 2 ' is completed, the 


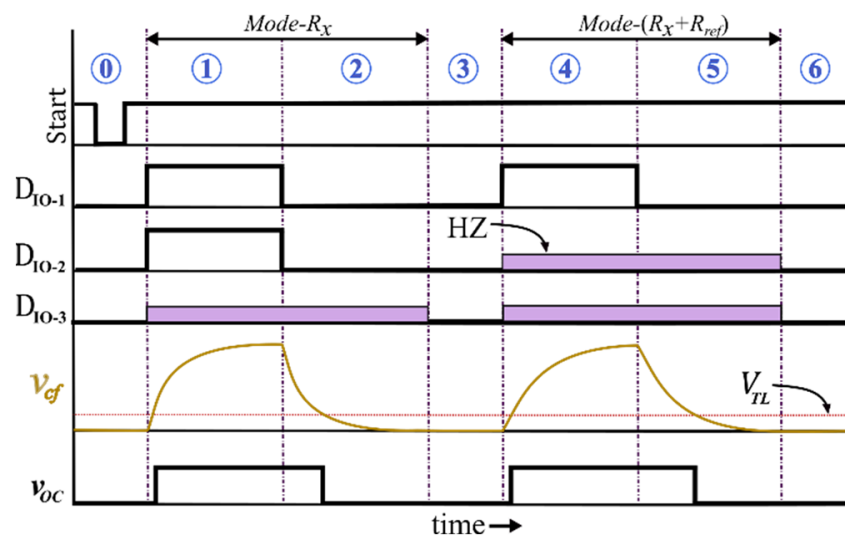

Fig. 2. Timing diagram of the proposed scheme.

$\mu \mathrm{C}$ sets $\mathrm{D}_{\mathrm{IO}-1}, \mathrm{D}_{\mathrm{IO}-2}$ and $\mathrm{D}_{\mathrm{IO}-3}$ once again to LOW (like in state ' 0 '). This is indicated as state ' 3 '. The next measurement is made in mode - $\left(R_{\text {ref }}+R_{x}\right)$. In this mode, the RC circuit has both $R_{x}$ and $R_{r e f}$ in series. In this mode, $\mathrm{D}_{\mathrm{IO}-2}$ is set to $\mathrm{HZ}$ throughout. $\mathrm{D}_{\mathrm{IO}-1}$ is set to HIGH to charge $C$ to $V_{C F}$ and LOW for it to discharge through the same path. These are executed through the states ' 4 ' and ' 5 ', respectively. This is illustrated in Fig. 2. The preset intervals for the states ' 4 ' and ' 5 ' are $T_{C h 2}$ and $T_{D h 2} . T_{C h 2}=T_{D h 2}$ and $T_{C h 2}, T_{D h 2}>>\left[\left(R_{x}+R_{r e f}\right) C_{e q}\right]$. As in mode - $R_{x}$, the time interval taken by $C$ to discharge from $V_{C F}$ to $V_{T L}$ is measured. The expressions for $V_{T L}$ and the corresponding discharge time, named $T_{2}$, in this mode are given by (4) and (5), respectively.

$$
\begin{aligned}
V_{T L} & =V_{C F} e^{-T_{2} /\left(R_{r e f}+R_{x}\right) C_{e q}} \\
T_{2} & =\left(R_{r e f}+R_{x}\right) C_{e q} \ln \left(\frac{V_{C F}}{V_{T L}}\right)
\end{aligned}
$$

Dividing (5) by (3),

$$
\frac{T_{2}}{T_{1}}=1+\frac{R_{r e f}}{R_{x}}
$$

Re-arranging (6),

$$
R_{x}=\frac{R_{\text {ref }}}{\left(T_{2}-T_{1}\right)} T_{1}
$$

Thus, the resistance $R_{x}$ of the capacitively-coupled resistive sensor element is determined in terms of the timer outputs $T_{1}$ and $T_{2}$, and the known resistor $R_{\text {ref }}$. State ' 5 ' completes onetime measurement of $R_{x}$. In case continuous measurements are to be made, the circuit operation enters state ' 6 ' once the discharge phase in mode - $\left(R_{r e f}+R_{x}\right)$ is completed. This state is the same as state ' 0 ' and facilitates discharge of any residual charges. Since $T_{1}$ and $T_{2}$ are outputs from the timer, the final output is digital. As can be seen from (7), the output does not depend on the values of the other parameters of the system such as $C_{1}, C_{2}, C, V_{C F}$ and $V_{T L}$, or any variations of those between two consecutive measurements of $R_{x}$.

\section{EXPERIMENTAL SET-UP AND RESULTS}

The hardware prototype of the proposed scheme, presented in Fig. 1, was realized in two steps. In the first step, a prototype was developed to test the feasibility of the approach without using a microcontroller. Instead, a data acquisition system (DAS), namely Analog Discovery kit (14-bit resolution, 100 MS/s sampling rate) from Digilent, and a Virtual Instrument (VI), developed using NI LabVIEW, were used as discussed in section III-A. The DAS has a function generator that was programmed to generate the HIGH and LOW state signal as required in the two modes of measurement given in Table-I and Fig. 2. In this DAS, the operation of the function generator is independent of the other operations, e.g., digitization, data transfer, etc. For the study, the signal at node ' $b$ ' was digitized using the DAS and analyzed as described below. In the second step, a microcontroller-based prototype was developed and tested. In this case, the two modes of measurement were realized by setting the microcontroller pins in accordance with Table-I and Fig. 2. The components used to build the prototypes are listed in Table - II.

Table II: PROTOTYPE

\begin{tabular}{cc||cc}
\hline \hline Component & Part/Value & Component & Part/Value \\
\hline \hline$V_{D D}$ & $5 \mathrm{~V}$ & $C_{1}, C_{2}$ & $4 \mathrm{nF}$ \\
Opamp & LF357 & $C$ & $1 \mathrm{nF}$ \\
microcontroller & ATMEGA328 & $R_{r e f}$ & $50.09 \mathrm{k} \Omega$ \\
\hline \hline
\end{tabular}

\section{A. Feasibility Study: Step-1}

In order to assess the practicality of the scheme, first an experimental set-up was developed in such way that its performance is not limited by the non-idealities of the microcontroller, particularly, the delay and input noise of the built-in comparator, the loading effect of the digital pin at node ' $b$ ', and the resolution of the built-in timer. For this, the functionality of the proposed circuit given in Fig. 1 was realized in hardware with the help of a DAS. First, the mode $R_{x}$ was realized. For this, the function generator was set to give a square wave of frequency $200 \mathrm{~Hz}$, with $5 \mathrm{~V}$ amplitude and an offset of $2.5 \mathrm{~V}$. This served the function of pin $\mathrm{D}_{\mathrm{IO}-1}$. Since this is mode - $R_{x}$ operation, $R_{\text {ref }}$ was shorted with a wire. Node 'b' was connected to the input of a voltage follower, built using a very low bias current opamp (LF357). Its output was given to the DAS. The analog channels of the DAS were used to acquire the important waveforms. The connections to the DAS were made such that channel-1 captured the signal at $\mathrm{D}_{\mathrm{IO}-1}$, whereas channel-2 captured $v_{c f}$ at the output of the voltage follower. To operate in mode - $\left(R_{x}+R_{\text {ref }}\right)$, the short across $R_{\text {ref }}$ was removed. The developed system enables performing mode - $R_{x}$ and mode - $\left(R_{x}+R_{\text {ref }}\right)$ independently and acquire the corresponding $v_{c f}$ waveforms.

A VI was accordingly developed, using LabVIEW, to acquire the signals by the DAS, and process them, as described further. For a chosen value of $R_{x}$, the $v_{c f}$ data during the discharge phase was analysed using the VI. The VI was programmed to perform an exponential fit of the $v_{c f}$ data and 


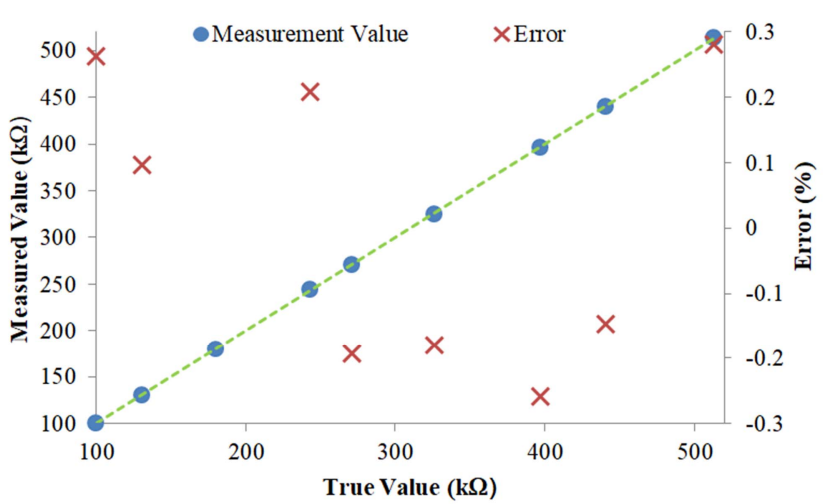

Fig. 3. Results from the prototype for a range of $R_{x}$. The output and the error (in percentage of true value) obtained are shown.

provide the parameters. These parameters, representing the decaying exponential curve, were used to compute the time $T_{1}$ using the VI. For this computation $V_{T L}=300 \mathrm{mV}$. The value of $V_{C F}$ according to the circuit elements used as in Table-II was $3.3 \mathrm{~V}$. Then the $v_{c f}$ data, for the discharge phase, corresponding to mode - $\left(R_{x}+R_{\text {ref }}\right)$ was taken and processed using the VI as detailed above. Following this, the value of $T_{2}$ for the same $V_{T L}$ was determined. Once $T_{1}$ and $T_{2}$ were computed, the value of $R_{x}$ was calculated by the VI using (7). This experiment was repeated for a range of $R_{x}$ from $100 \mathrm{k} \Omega$ to $500 \mathrm{k} \Omega$, in several steps. In each measurement, the actual value of $R_{x}$ was measured using a 6-1/2 digit multimeter, 34410A, from Agilent Technologies. This was used to compute the error introduced by the proposed method. The results are shown in Fig. 3. The maximum error noted during the experiments was $0.28 \%$, indicating the high accuracy of the proposed approach.

\section{B. Feasibility Study: Step-2}

In this experiment, instead of using the DAS and the VI as in step-1, the scheme was entirely realized using a microcontroller as given in Fig. 1. The output of the voltage follower with voltage $v_{c f}$ was directly given to node $\mathrm{A}_{1}$ of the microcontroller, and the built-in timer was programmed to measure the time $T_{1}$ and $T_{2}$ during the corresponding states ' 2 ' and ' 5 ', respectively, as given in Table-I. Then, this experiment was repeated for the same set of values of $R_{x}$ employed in section III-A. The value of $R_{x}$, in each case, and

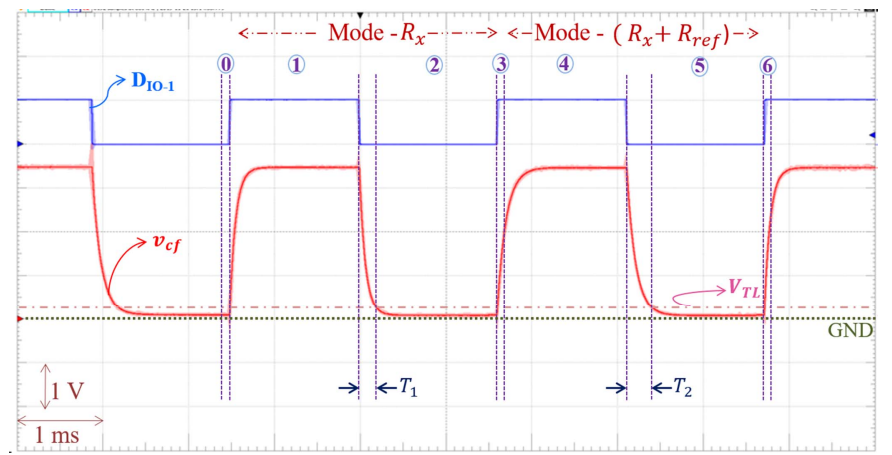

Fig. 4. Observed waveforms of charging and discharging cycles, from the prototype, for the states ' 0 ' to ' 6 '.

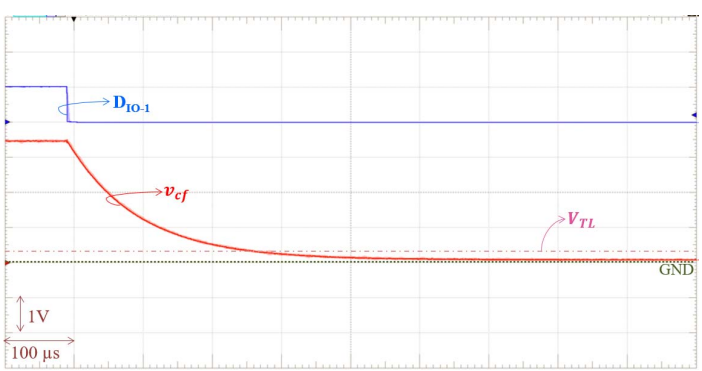

Fig. 5. $v_{c f}$ recorded during the discharge phase of mode $-\left(R_{x}+R_{r e f}\right)$.

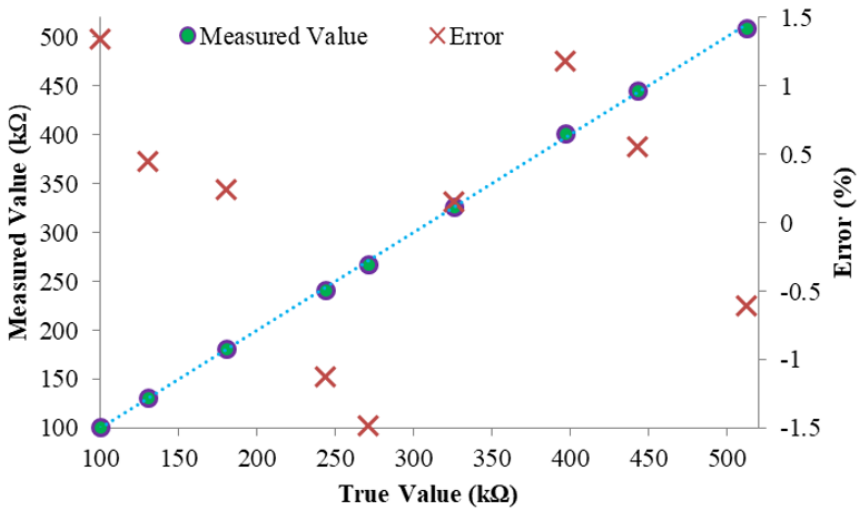

Fig. 6. Results from the prototype- 2 for a range of $R_{x}$. This prototype was implemented using microcontroller.

the corresponding error was computed. The important waveforms were observed using the oscilloscope in Analog Discovery kit, for the charge and discharge cycle for mode - $R_{x}$ followed by mode - $\left(R_{x}+R_{\text {ref }}\right)$. A screenshot of the same is given in Fig. 4. A zoomed-in version of the discharge phase alone for mode - $\left(R_{x}+R_{\text {ref }}\right)$ is given in Fig. 5. The results obtained are presented in Fig. 6. The maximum error noted during the experiments was $1.48 \%$. The output of this experiment, in comparison with that from section III-A, reflects the effect of the non-idealities of the components and ICs used in the prototype. To achieve higher accuracy, one could use a microcontroller with higher time resolution, and a comparator with lesser delay and noise, which will be studied in the future work. Results from the feasibility studies prove the practicality of the proposed direct microcontroller approach.

\section{CONCLUSION}

A direct microcontroller interface for capacitivelycoupled resistive sensor has been presented in this paper. Such a scheme for the measurement of resistance in a capacitivelycoupled resistive sensor has been attempted for the first time. Since no active electronic components are used between the sensor and the microcontroller, the complexity, size and power consumption of the measurement system are considerably reduced. The output of the proffered scheme is independent of the values of the coupling capacitors, the charging capacitor, the dc excitation voltage, and the preset threshold voltage. It is simple in design and completes measurement in a few 
milliseconds. The feasibility study of the proposed circuit exhibits a maximum error of $0.28 \%$, when evaluated without considering the non-idealities of the microcontroller, and $1.48 \%$, otherwise. Higher accuracy can be achieved by selecting a microcontroller with more appropriate specifications. This scheme does not use any expensive parts and can hence realize a low-cost measurement system. Due to these features, the proposed measurement scheme is expected to be extensively used in non-intrusive sensing and monitoring applications based on capacitively-coupled resistive sensors.

\section{ACKNOWLEDGMENT}

The authors would like to thank Department of Science and Technology (DST), Govt. of India, for its financial assistance (Grant Number SERB/F/4573/2016-17) in carrying out the research activities presented in this paper.

\section{REFERENCES}

[1] L. Pan, A. Chortos, G. Yu, S. Isaacson, R. Allen, Y. Shi, R. Dauskardt and Z. Bao, "An ultra-sensitive resistive pressure sensor based on hollow-sphere microstructure induced elasticity in conducting polymer film," Nature Commun., vol. 5, pp. 3002, Jan. 2014

[2] L. Paredes-Madrid, J. Fonseca, A. Matute, E. Gutiérrez Velásquez and C. Palacio, "Self-Compensated Driving Circuit for Reducing Drift and Hysteresis in Force Sensing Resistors," Electronics, Aug 2018.

[3] A. Song, L. Fu, "Multi-dimensional force sensor for haptic interaction: A review," Virtual Reality Intell. Hardw., vol. 1, no. 2, pp. 121-135, Jan. 2019.

[4] E. O. Doebelin, Measurement Systems-Application and Design, 5th ed. New York, NY, USA: McGraw-Hill, 2004

[5] D. Fourie, "Touch surface having capacitive and resistive sensors," U.S. Patent application US 14/143,212., Jul. 2, 2015.

[6] Y. Xue, Z. Ju, K. Xiang, J. Chen and H. Liu, "Multimodal Human Hand Motion Sensing and Analysis - A Review," in IEEE Transactions on Cognitive and Developmental Systems, vol. 11, no. 2, pp. 162-175, June 2019.

[7] Á. Pintér, I. Dénes, "Interface circuit for measuring small capacitance changes in sensor networks," IET Science, Measurement \& Technology, vol. 9, no. 5, pp. 570-578, July 2015.
[8] L. Areekath, B. George and F. Reverter, "An Extended Study on an Interference-Insensitive Switched Capacitor CDC," IEEE Sensors Journal, vol. 19, no. 18, pp. 8283-8292, Sept.15, 2019.

[9] Y. Xue, Z. Ju, K. Xiang, J. Chen and H. Liu, "Multimodal Human Hand Motion Sensing and Analysis-A Review," IEEE Trans. Cogn. Devel. Syst., vol. 11, no. 2, pp. 162-175, June 2019.

[10] S. Chakraborty, S. K. Bera, N. Mandal, S. C. Bera, "Study on further modification of non-contact capacitance type-level transducer for a conducting liquid, " IEEE Sensors J., vol. 15, no. 11, pp. 6678-6688, Nov. 2015.

[11] G. Mattana, T. Kinkeldei, D. Leuenberger, C. Ataman, J. J. Ruan, F. Molina-Lopez, A.V. Quintero, G. Nisato, G. Tröster, D. Briand and N.F. de Rooij, "Woven temperature and humidity sensors on flexible plastic substrates for e-textile applications, " IEEE Sensors J., vol. 13, no. 10, pp. 3901-3909, Oct. 2013.

[12] K. K.Tejaswini, B. George, V. J. Kumar, R. Srinivasan and S. Tata, "A Capacitive Coupled Non-Contact Probe for the Measurement of Conductivity of Liquids, " IEEE Trans. Instrum. Meas., vol. 68, no. 5, pp. 1602-1610, May 2019.

[13] S. L. Schiefelbein, N. A. Fried, K. G. Rhoads and D. R. Sadoway, "A high-accuracy, calibration-free technique for measuring the electrical conductivity of liquids," Review of scientific instruments, vol. 28, no. 6 , pp. 1141-1149, Dec. 1998.

[14] E. Sifuentes, R. Gonzalez-Landaeta, J. Cota-Ruiz, and F. Reverter, "Seat occupancy detection based on a low-power microcontroller and a single FSR, " Sensors, vol. 19, no. 3, 699 (11pp), Feb. 2019

[15] R. Anandanatarajan, U. Mangalanathan, and U. Gandhi, "Enhanced microcontroller interface of resistive sensors through resistance-to-time converter, " IEEE Trans. Instrum. Meas. (in press).

[16] F. Reverter and Ò. Casas, "Interfacing differential capacitive sensors to microcontrollers: a direct approach," IEEE Trans. Instrum. Meas., vol. 59, no. 10, pp. 2763-2769, Oct. 2010.

[17] N. Ramadoss, and B. George, "A simple microcontroller based digitizer for differential inductive sensors," Proc. of 2015 IEEE International Instrumentation and Measurement Technology Conference (I2MTC), May 11, 2015, pp. 148-153.

[18] Z. Kokolanski, J. Jordana, M. Gasulla, V. Dimcev, and F. Reverter, "Direct inductive sensor-to-microcontroller interface circuit," Sens. Actuators A Phys., vol. 224, pp. 185-191, 2015

[19] J.A. Hidalgo-López, J.A. Botín-Córdoba, J.A. Sánchez-Durán and O.Oballe-Peinado, "Fast calibration methods for resistive sensor readout based on direct interface circuits, " Sensors, vol. 19, no. 18, 3871 (20pp), Sep. 2019. 